\title{
Gene expression during induced differentiation of sheep bone marrow mesenchymal stem cells into osteoblasts
}

\author{
Y. Hu, X.-X. Tang and H.-Y. He \\ Department of Prosthodontics, \\ First Affiliated Hospital of Xinjiang Medical University, Xinjiang, China \\ Corresponding author: H.-Y. He \\ E-mail: txx_hy@yeah.net
}

Genet. Mol. Res. 12 (4): 6527-6534 (2013)

Received October 5, 2012

Accepted September 20, 2013

Published December 11, 2013

DOI http://dx.doi.org/10.4238/2013.December.11.4

\begin{abstract}
The aim of this study was to observe the dynamic expression of osteopontin, type I collagen, and osteocalcin genes during the differentiation of bone marrow mesenchymal stem cells (BMSCs) into osteoblasts and confirm whether BMSCs can differentiate and mature into osteoblasts. A healthy, 2-month-old Altay tailed sheep was obtained, and $10 \mathrm{~mL}$ bone marrow was extracted from the posterior superior iliac spine of the sheep via puncture. Sheep BMSCs were extracted using a whole bone marrow culture method and cultured for osteogenic induction. Total RNA from the BMSCs was extracted, and the gene expression levels of osteopontin, type I collagen, and osteocalcin at the 1st, 2nd, 3rd, and 4th passages of uninduced and induced BMSCs were detected using reverse transcription-polymerase chain reaction. The surface antigen CD44 was also detected in the BMSCs. After induction by culturing in osteoinductive medium, BMSCs in the 1st, 2nd, 3rd, and 4th passages displayed stagespecific expression of osteopontin, type I collagen, and osteocalcin genes. The positive expression rate of BMSC-specific antigen CD44 in the 4th passage of osteogenic-induced BMSCs was 99.8\%. In vitro-cultured
\end{abstract}


sheep BMSCs in osteogenic culture medium gradually differentiated into osteoblasts, which stage-specifically expressed osteoblast-specific genes such as osteopontin, type I collagen, and osteocalcin at the 4th passage after induction. At this passage, these cells had osteoblast functions.

Key words: Osteoblasts; Gene expression; Induced differentiation; Bone marrow mesenchymal stem cells; Sodium $\beta$-glycerophosphate

\section{INTRODUCTION}

Bone marrow mesenchymal stem cells (BMSCs) are multipotent stromal cells derived from the mesoderm. They are mainly present in the connective tissues and organ interstitium and are most abundant in bone marrow tissues but can also be isolated from fetal cord blood (Erices et al., 2000). Under certain induction conditions, BMSCs can differentiate into mesodermal cells such as osteoblasts, chondrocytes, adipocytes, and stromal cells. BMSCs also have low immunogenicity characteristics and immunomodulatory functions that can reduce the immune rejection that occurs after transplantation. Because they are easy to obtain and have strong osteogenic differentiation capabilities, BMSCs are often used as seeder cells for bone tissue engineering and as vector cells for gene therapy (Studeny et al., 2002; Potapova et al., 2004). Currently, the dynamic gene expression patterns that occur during the differentiation of BMSCs into osteoblasts remain unclear. Studies have shown that osteogenic-induced BMSCs express non-osteoblast-specific genes such as osteocalcin, osteopontin, and type I collagen. However, few studies have pursued the spatial and temporal sequences of osteocalcin, osteopontin, and type I collagen expression at the 1st, 2nd, 3rd, and 4th passages of BMSCs after osteogenic induction.

This study used a whole bone marrow adherence culture method to isolate and culture sheep BMSCs. Then, osteogenic induction on the 1st, 2nd, 3rd, and 4th passages of cells was performed to observe the cell morphology changes of BMSCs during their differentiation into osteoblasts and to detect the expression of non-osteoblast-specific genes such as osteocalcin, osteopontin, and type I collagen. The positive rate of a BMSC antigen, CD44, was measured. The growth curve was measured using the 3-(4,5-dimethylthiazol-2-yl)-2,5-diphenyltetrazolium bromide (MTT) assay. The culture conditions, proliferation activity, expression of osteoblast-specific genes, and functional status of sheep BMSCs during differentiation into osteoblasts were also explored.

\section{MATERIAL AND METHODS}

\section{Experimental animal}

The experimental animal was a healthy, male, 2-month-old Altay tailed sheep with a body weight of $6 \mathrm{~kg}$. The experimental animal use permit No. was A-20101020026, and the animal was provided by the First Affiliated Hospital of Xinjiang Medical University.

\section{Isolation and primary culture of sheep BMSCs}

The Altay tailed sheep was anesthetized via intramuscular injection of $0.9 \mathrm{~mL} 10 \%$ 
chloral hydrate (v/v) and $0.6 \mathrm{~mL}$ diazepam. The limbs were immobilized, the surgical area was shaved, and routine disinfection and draping were performed. A bone marrow cavity puncture was performed at the posterior superior iliac spine. Ten millimeters of bone marrow was collected and placed separately in A and B centrifuge tubes containing $200 \mathrm{U} / \mathrm{mL}$ heparin. After through mixing, the $5 \mathrm{~mL}$ bone marrow in each tube was filtered through 200-mesh filters, diluted with 10-mL Dulbecco's modified Eagle medium/F12 (10\% fetal bovine serum, v/v), and inoculated into $25-\mathrm{cm}^{2}$ culture flasks. After 3 days, half of the culture medium was replaced. All of the medium was replaced after 7 days, and then the medium was replaced every 3 days. The cells were passaged at a 1:3 ratio when they were $80-90 \%$ confluent. The vitality and growth of the cells were observed every day using an inverted fluorescence microscope.

\section{Flow cytometric identification}

Cells from the 2 groups at the 4th passage after induction were collected and they were digested using $2.5 \mathrm{~g} / \mathrm{L}$ trypsin. After centrifugation, the cells were washed with phosphate-buffered saline (PBS) 3 times, and the cell concentration was adjusted to $1 \times 10^{6}$ cells/ $\mathrm{mL}$. Fluorescence-labeled CD44 antibodies were added to each tube and incubated for $20 \mathrm{~min}$ in darkness. The unbound antibodies were washed off using PBS, and cell surface antigens were detected using a flow cytometer. Immunoglobulin G1 was added into the culture of the 4th passage of non-induced culture cells as a negative control.

\section{Differentiation of induced cultured BMSCs into osteoblasts}

The second passage of BMSCs (inoculation concentration, 1 x $10^{6} \mathrm{cells} / \mathrm{L}$ ) was cultured in mineralization osteoinductive culture medium $(10 \% \mathrm{v} / \mathrm{v}$ fetal bovine serum, Dulbecco's modified Eagle medium/F12, $1 \times 10^{-8} \mathrm{mM}$ dexamethasone, $0.01 \mathrm{mM}$ sodium $\beta$-glycerophosphate, $0.05 \mathrm{~g} / \mathrm{L}$ vitamin $\mathrm{C}, 100 \mathrm{U} / \mathrm{L}$ penicillin, and $100 \mathrm{U} / \mathrm{L}$ streptomycin). The medium was changed every 3 days, and the cells were passaged when they became $80-90 \%$ confluent.

\section{Detection of BMSC growth curve}

Cells from the 2 groups at the 3rd, 5th, and 7th passages were inoculated onto 96-well plates at $5 \times 10^{4} \mathrm{cell} / \mathrm{mL}, 200 \mu \mathrm{L} /$ well, and 5 wells per group. After inoculation, $20 \mu \mathrm{L}$ MTT solution (MTT was dissolved in PBS at a concentration of $5 \mathrm{~g} / \mathrm{L}$, filter-sterilized, and stored at $4{ }^{\circ} \mathrm{C}$ in darkness) was added to the wells every $24 \mathrm{~h}$ and incubated at $37^{\circ} \mathrm{C}$ for $4 \mathrm{~h}$. After the culture medium was discarded, $150 \mu \mathrm{L}$ dimethyl sulfoxide was added and vortexed for 10 min to dissolve the crystals fully. Optical density (OD) values were measured at $490 \mathrm{~nm}$. An average OD value of 5 wells in each group was used to represent the growth condition of the 2 groups of cells. The growth curve of the cells was plotted using time as the horizontal axis and the OD value as the longitudinal axis.

\section{Total RNA extraction and reverse transcription-polymerase chain reaction (PCR)}

Total RNA was extracted with TRIzol(Beijing, Bioteck, China). The primers were designed using the Primer 5.0 software and synthesized by Shanghai ShengGong Company 
(China). The primer sequences are shown in Table 1. The 50- $\mu \mathrm{L}$ sample of the PCR system included $25 \mu \mathrm{L}$ of Premix Ex Taq (Beijing, Bioteck, China), $2 \mu \mathrm{L}$ template complementary DNA, $1.0 \mu \mathrm{L}$ forward and reverse primers $(20 \mu \mathrm{M})$, and double-distilled water.

Table 1. Primers used in this study.

\begin{tabular}{llc}
\hline Gene & Primers & Products \\
\hline OPN & Sense: 5'-AGGGACAGAACTCGGCCGTG-3' & 259 bp \\
& Anti-sense: 5'-CCAGTGTATTGTGTTGGCCCTC-3' & 394 bp \\
OC & Sense: 5'-CCCCATGCTCGCCCTGCTGG-3' & 290 bp \\
& Anti-sense: 5'-TCTGGAGTTTATCTAGAGCAGCG-3' \\
Collagen-I & Sense: 5'-ACCTACCACTGCAAGAACAGCG-3' & \\
& Anti-sense: 5'-AAGCAGACAGAGCCGATGTCG-3' \\
\hline
\end{tabular}

\section{RESULTS}

\section{Observation of growth and morphology of sheep BMSCs}

Cells obtained with the whole bone marrow culture method displayed a scattered distribution at the bottom of the culture flasks, and they were mixed with bone marrow hematopoietic cells. A majority of the suspension cells could be removed after half of the medium was replaced the 3rd day after induction; the suspension cells were essentially all removed when the medium was changed after day 7 . In the culture flask, some cells were adnately scattered, and the morphology of the cells showed spindles, triangles, and polygons. After 8 days, the number of adherent cells significantly increased, the number of cell divisions increased, and many swirl-shaped cell colonies were visible. The distribution of the cells was uneven, and many cells had 2 or 3 protrusions (Figure 1A). The morphology of the induced BMSCs changed from long spindles into squares and polygons, and the cell volume increased (Figure 1B).

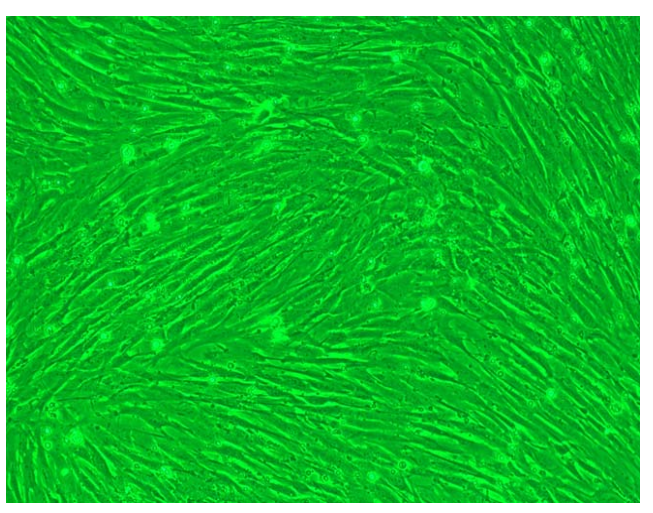

A

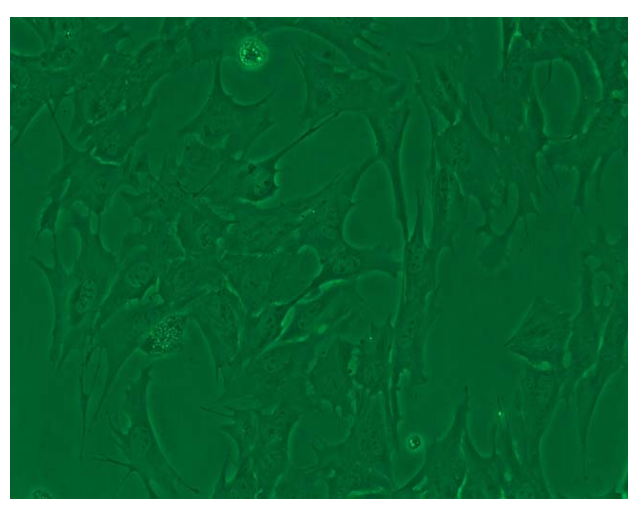

B

Figure 1. A. Non-induced culture BMSCs (100 X); B. induced apoptosis in cultured BMSCs (200 X).

\section{Growth curve of sheep BMSCs}

The MTT detection results of induced BMSCs showed that the induced cells at the 
third passage proliferated slowly the first $72 \mathrm{~h}$ after inoculation. After $96 \mathrm{~h}$, the cells started to proliferate rapidly and entered the exponential growth phase. The proliferation of cells significantly decreased after $216 \mathrm{~h}$, and the cells entered the plateau phase. Compared with that of the uninduced BMSCs, the proliferation of the induced BMSCs decreased (Figure 2).

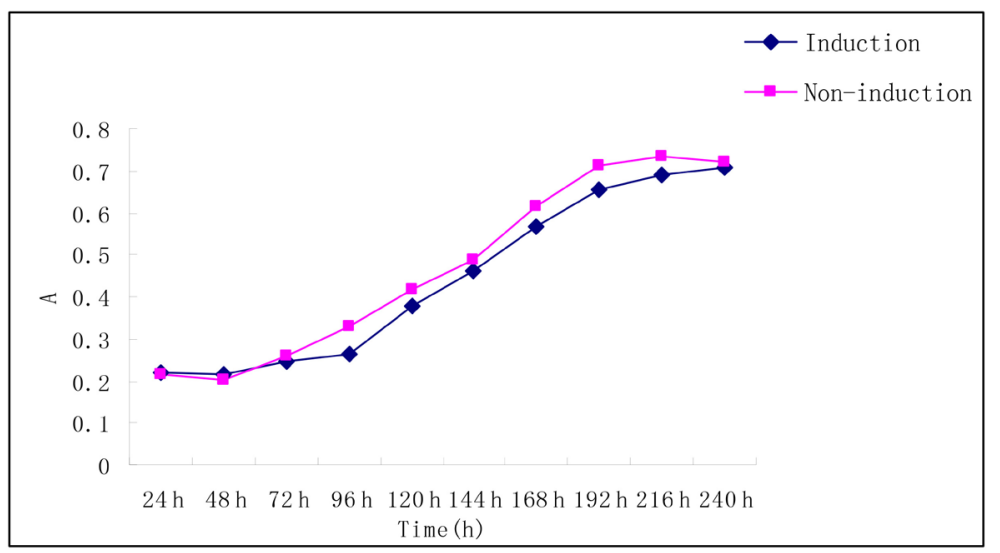

Figure 2. BMSCs growth curve of uninduced and induced group.

\section{Detection of sheep BMSC surface antigens}

Flow cytometric results showed that the CD44-positive rates of induced and uninduced cells at the 4th passage were 99.8 and $95.4 \%$, respectively (Figure $3 \mathrm{~A}, \mathrm{~B}$ ), whereas the positive rate in the negative control cells was $0.6 \%$ (Figure $3 \mathrm{C}$ ).

A

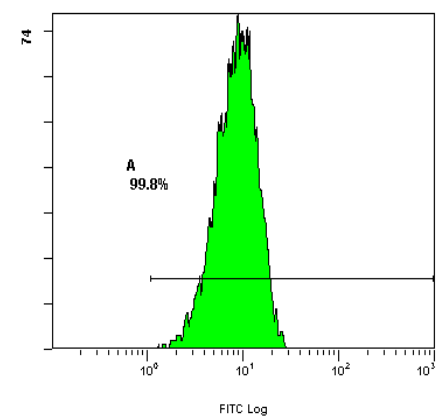

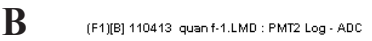

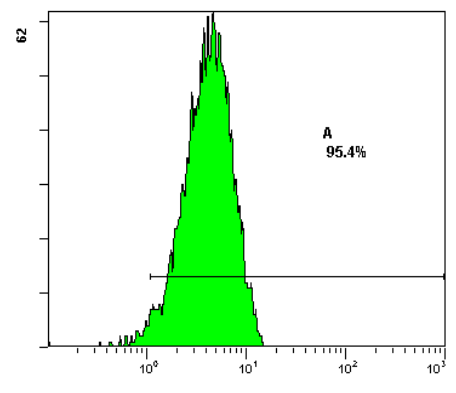

FITC Log
C

[F1][B] 110330 IgG-quan f.LMD : PMT2 Log - ADC

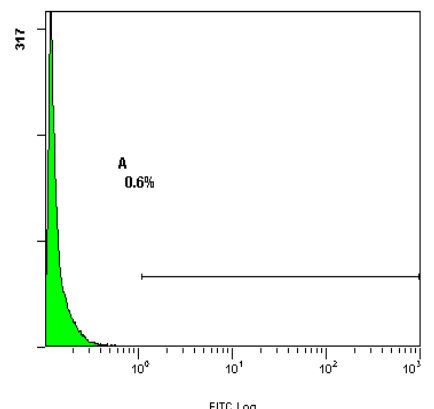

Figure 3. A. CD44-positive rate of 4th generation induced apoptosis cells; B. CD44-positive rate of 4th generation uninduced apoptosis cells; C. CD44-positive rate of control.

\section{Detection of genes in BMSCs during in vitro osteogenic differentiation}

During the process of osteogenic differentiation of BMSCs, the expression of mes- 
senger RNAs (mRNAs) for osteopontin, osteocalcin, and type I collagen occurred in a specific order. The induced BMSCs at the 1st passage expressed type I collagen. The induced BMSCs at the 2nd passage expressed type I collagen and osteopontin, and the induced BMSCs at the 3rd and 4th passages expressed type I collagen, osteocalcin, and osteopontin. The uninduced BMSCs at the 1st passage in the control group expressed type I collagen and osteopontin, whereas uninduced cells at the $2 \mathrm{nd}, 3 \mathrm{rd}$, and 4th passages expressed only type I collagen.

The PCR product of the osteopontin gene was approximately $259 \mathrm{bp}$, and the agarose gel electrophoresis results showed that the size of the target fragment was consistent with the marker. The gene fragment of osteocalcin was approximately $394 \mathrm{bp}$ and consistent with the marker. The gene fragment of type I collagen was approximately $290 \mathrm{bp}$ and consistent with the marker. The sequencing results showed that osteopontin, osteocalcin, and type I collagen had individual nucleotide mutations but contained no frameshift mutations. The electropherogram results are shown in Figure 4A-C.

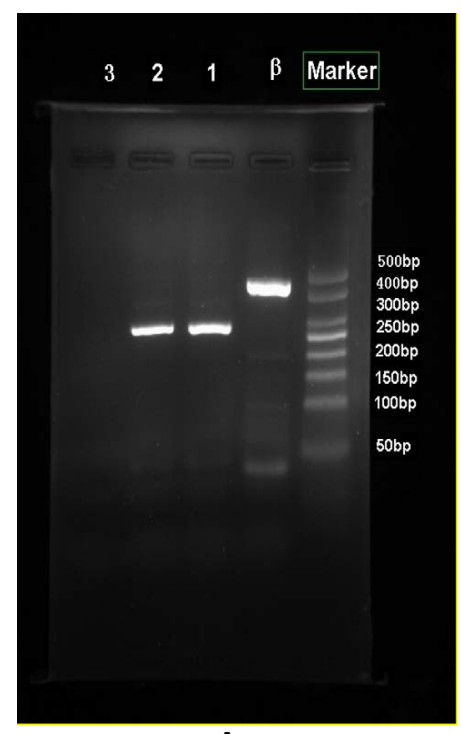

A

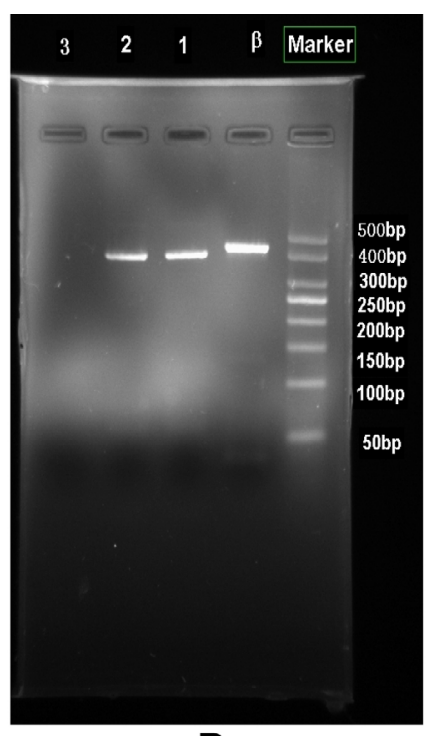

$\mathrm{B}$

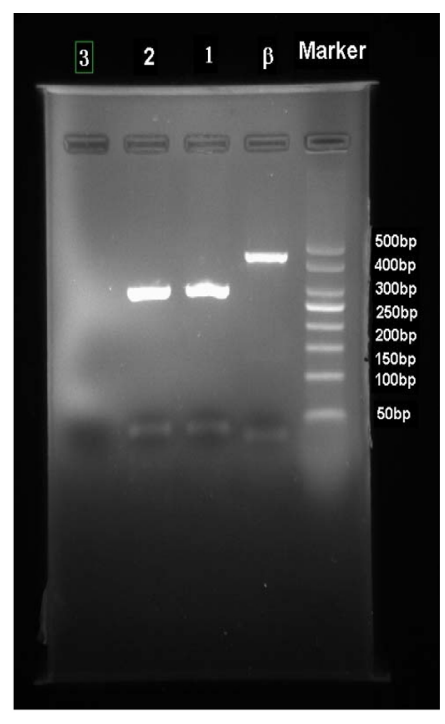

C

Figure 4. A. Electrophoresis of osteopontin gene. Lane $\beta=\beta$-actin; lanes 1 and $2=$ OPN gene; lane $3=$ negative control. B. Electrophoresis of osteocalcin gene. Lane $\beta=\beta$-actin; lanes 1 and 2: OC gene; lane $3=$ negative control. C. Electrophoresis of type I collagen gene. Lane $\beta=\beta$-actin; lanes 1 and $2=$ type I collagen gene gene; lane $3=$ negative control.

\section{DISCUSSION}

BMSCs are believed to have multiple antigen markers and exhibit non-uniqueness; thus, a specific antigen marker for BMSCs remains to be found (Pittenger et al., 1999; Zhu et al., 2004; Delorme et al., 2006; Stolzing and Scutt, 2006; Sun et al., 2010). BMSCs and hematopoietic stem cells coexist in the bone marrow. BMSCs express no hematopoietic stem cell surface antigens such as hematopoietic stem cell marker antigen CD34 or white blood cell marker antigen CD45, but they do express adhesion molecules, growth factor receptors, and integrin family members, 
such as CD29, CD44, CD90, CD105, and CD166, of which CD44 is considered an important antigen marker of BMSCs. In this study, flow cytometry was used to screen for BMSC-specific antigen CD44. The results showed that the CD44-positive rate was $99.8 \%$ in induced cells at the 4th passage, $95.4 \%$ in uninduced cells at the 4 th passage, and $0.7 \%$ in cells of the negative control group. With a gradual increase in the number of medium changes and passages, the confounding hematopoietic lineage cells were gradually removed, and the cultured BMSCs in the experimental groups continued to proliferate; thus, the CD44-positive rate increased. This result shows that the purity of the isolated and cultured BMSCs in this experiment was high, confirming that the cells were derived from differentiated BMSCs after induction.

BMSCs can undergo osteoinduction in vitro; their differentiation and maturation into osteoblasts have 3 major stages: cell proliferation, matrix maturation, and mineralization. The differentiation process includes directional differentiation of multipotent stromal cells into preosteoblasts, immature osteoblasts, and mature osteoblasts (Cotter et al., 2008). The osteoblast cells predominantly express extracellular matrix proteins such as alkaline phosphatase, osteocalcin, osteopontin, and type I collagen during the cell proliferation, matrix maturation, and mineralization phases. Observation of the mRNA expression levels of these marker genes helps to some extent to distinguish the stages of differentiation and maturation of BMSCs into osteoblasts. Current studies have shown that type I collagen, osteopontin, and alkaline phosphatase are secreted during the early stage of osteogenic differentiation, whereas osteocalcin, a marker of a mature osteoblast differentiation, is a necessary factor for bone calcification and mineralization. After 1 week of culture, the expression levels of these genes usually increase (Balcerzak et al., 2003; Janssens et al., 2005; Osyczka and Leboy, 2005). This study showed that days 3-6 in sheep BMSC osteogenic induction culture constituted the cell proliferation phase, days 6-14 composed the matrix maturation phase, and days 14-21 made up the mineralization phase. Therefore, the results revealed the expression mechanism of some nonspecific genes during the process of the induced differentiation of BMSCs into osteoblasts. The results also have important implications for the identification of the differentiation of BMSCs into osteoblasts, for the maturation status of BMSCs as they differentiate, and for the assessment of osteogenic functions.

This study showed that the 1st passage of cultured, uninduced BMSCs expressed type I collagen and osteopontin, but cells at the $2 \mathrm{nd}, 3 \mathrm{rd}$, and 4 th passages expressed only type I collagen. The induced BMSC culture at the 1st, 2nd, 3rd, and 4th passages did not express osteopontin, possibly because BMSCs at the 1st passage retained a small portion of hematopoietic stem cells, confounding cells, and some growth factors (Tang et al., 2005). With an increase in the number of medium changes, this small portion was removed; thus, the cultured BMSCs no longer expressed osteopontin.

This study also showed that during osteogenic induction culture, induced BMSCs expressed type I collagen at the 1st passage, type I collagen and osteopontin at the 2nd passage, and type I collagen, osteocalcin, and osteopontin at the 3rd and 4th passages. During the process of induced differentiation of BMSCs into osteoblasts, with prolonged induction time, the 1st passage of BMSCs in the cell proliferation phase expressed type I collagen, the 2nd passage of BMSCs in the matrix maturation phase started to express osteopontin, and the 3rd and 4th passages of BMSCs in the mineralization phase expressed osteocalcin. These results indicated that BMSCs successfully differentiated and matured into osteoblasts, which was consistent with results reported by Balcerzak et al. (2003).

The stage-specific expression of collagen, osteopontin, and osteocalcin mRNAs showed 
that BMSCs were differentiated into proliferation, matrix maturation, and mineralization phases. These results revealed the mechanism of expression of some genes during the osteogenicinduced differentiation of BMSCs. The induced BMSCs had certain osteogenic functions and potential, and they are expected to be used as ideal seed cells for bone tissue engineering.

\section{ACKNOWLEDGMENTS}

Research supported financially by the National Natural Science Foundation of China (\#81060088), the Autonomous Region Natural Science Genome Project (\#2011211A073), and the Xinjiang Medical Graduate Innovation Fund (\#MC2010-8).

\section{REFERENCES}

Balcerzak M, Hamade E, Zhang L, Pikula S, et al. (2003). The roles of annexins and alkaline phosphatase in mineralization process. Acta Biochim. Pol. 50: 1019-1038.

Cotter EJ, Ip HS, Powderly WG and Doran PP (2008). Mechanism of HIV protein induced modulation of mesenchymal stem cell osteogenic differentiation. BMC Musculoskelet. Disord. 9: 33.

Delorme B, Chateauvieux S and Charbord P (2006). The concept of mesenchymal stem cells. Regen. Med. 1: 497-509.

Erices A, Conget P and Minguell JJ (2000). Mesenchymal progenitor cells in human umbilical cord blood. Br. J. Haematol. 109: 235-242.

Janssens K, ten DP, Janssens S and Van HW (2005). Transforming growth factor-beta1 to the bone. Endocr. Rev. 26: 743-774.

Osyczka AM and Leboy PS (2005). Bone morphogenetic protein regulation of early osteoblast genes in human marrow stromal cells is mediated by extracellular signal-regulated kinase and phosphatidylinositol 3-kinase signaling. Endocrinology 146: 3428-3437.

Pittenger MF, Mackay AM, Beck SC, Jaiswal RK, et al. (1999). Multilineage potential of adult human mesenchymal stem cells. Science 284: 143-147.

Potapova I, Plotnikov A, Lu Z, Danilo P, Jr., et al. (2004). Human mesenchymal stem cells as a gene delivery system to create cardiac pacemakers. Circ. Res. 94: 952-959.

Stolzing A and Scutt A (2006). Age-related impairment of mesenchymal progenitor cell function. Aging. Cell 5: 213-224.

Studeny M, Marini FC, Champlin RE, Zompetta C, et al. (2002). Bone marrow-derived mesenchymal stem cells as vehicles for interferon-beta delivery into tumors. Cancer Res. 62: 3603-3608.

Sun K, Wang GB and Zu Y (2010). Different age children bone marrow mesenchymal stem cells from autologous serum in vitro culture and into bone differentiation. Chin. Tissue Eng. Res. Clin. Rehabil. 14: 7425-7429.

Tang XX, He HY, Xu HF, Hu Y, et al. (2011). Effects of two separation methods on biological characteristics of sheep bone marrow mesenchymal stem cells. Zhongguo Zuzhi Gongcheng Yanjiu yu Linchuang Kangfu. 15: 9137-9140

Zhu H, Xu JZ and Zhou Q (2004). Different ages of human bone marrow mesenchymal stem cell proliferation and osteogenic differentiation study. Chin. J. Orthop. Surg. 12: 759-761. 